\title{
The influence of social processes on the timing of cancer diagnosis: a research agenda
}

\author{
Jessica Corner, Lucy Brindle
}

Faculty of Health Sciences, University of Southampton, Southampton, UK

\section{Correspondence to} Professor Jessica Corner, Faculty of Health Sciences, Building 67, University of Southampton, Southampton S017 1BJ, UK:

j.l.corner@soton.ac.uk

Accepted 14 October 2010

\begin{abstract}
This paper sets out to review the influence of social processes on the timing of the diagnosis of cancer and to explore the potential for promoting earlier diagnosis by addressing social factors that influence symptom recognition and the diagnostic process. Social processes refer to the means by which culture and social organisation may impact on timely cancer diagnosis. The paper calls for concerted action around an important and developing research agenda that may prove highly valuable in the quest to secure prompt diagnosis for cancer and through it improved outcomes for individuals.
\end{abstract}

Cancer survival rates have improved dramatically over the past 30 years although they remain stubbornly poor among certain groups such as patients with lung and pancreatic cancer, the socioeconomically deprived and minority ethnic groups. ${ }^{12}$ Survival rates for cancer in the UK show improvement, but remain lower than in other European countries. ${ }^{3}{ }^{4}$ Now that much has been done to address health system performance and ensure that treatment pathways are optimised, it is recognised that late diagnosis is an important factor in cancer outcomes. The recently launched UK National Awareness and Early Detection Initiative (NAEDI) seeks to address this. ${ }^{5}$ However, the knowledge base or evidence to support such an initiative remains limited.

Analysis of cancer survival data across Europe has been undertaken to identify the relative contribution that prompt or early diagnosis makes to the better survival outcomes achieved in certain European countries, notably Sweden, France, Italy, Belgium and Switzerland and the poor relative outcomes in the UK. ${ }^{6}$ These studies have used 1-year survival rates as a proxy measure for early diagnosis as it is indicative of disease stage at diagnosis. Results of the analysis indicate that for certain cancers, notably breast and cervical cancers, poor survival in the first year after diagnosis accounts for the relatively poor outcomes from the disease compared with other countries despite the existence of screening programmes. These poor survival rates may be explained by small subgroups of women diagnosed with late stage disease not being detected by screening. For other cancers, such as lung cancer, there is poor relative 1-year and 5 -year survival, suggesting that both late diagnosis and access to optimum treatment and care may account for the differences. ${ }^{7}$ Variation in screening coverage and participation rates across Europe might account for some of the variation in survival outcomes. However, even when there are well organised national screening programmes with high participation, many tumours are diagnosed following symptomatic presentation rather than screening. ${ }^{8}$ Analyses of cancer survival data have provided greater understanding as to the extent of late diagnosis and its contribution to outcomes in cancer survival, and a recognition that focusing on promoting earlier diagnosis, alongside and in addition to cancer screening programmes, needs to be given greater emphasis.

These analyses have led to an emergent science about what promotes or inhibits timely (early) diagnosis of cancer as well as developing a deeper understanding of the dynamic around the processes of becoming diagnosed with cancer and the relationship between promoting earlier diagnosis with improved outcomes for the disease. Emergent concepts, informed by social and psychological sciences as well as clinical science, are influencing policy development and research agendas. These are delayed diagnosis, cancer awareness and lay and professional symptom recognition. In each of these areas work is underway or has been completed to develop insights into the contribution each might make to cancer survival outcomes and to investigate potential intervention strategies to promote earlier diagnosis.

\section{DELAY}

Burgess et $a l^{9}$ identify three types of delay in the diagnosis of cancer: first, that generated by the individual who fails to act on suspicious symptoms through fear, lack of knowledge or because they do not interpret their symptoms as being serious. Second, doctor or practitioner-generated delay that occurs through misinterpreting symptoms or not initiating referral for diagnostic tests. Third, system or hospital-generated delay that occurs through inefficiency, or long waiting times for appointments or tests. These three types of delay have been operationalised for measurement purposes in studies as patient delay (the time between first noticing symptoms and first consulting a doctor), practitioner delay (the interval between first consultation and referral) and hospital delay (the time between referral and diagnosis). ${ }^{10}$ A growing number of studies has attempted to determine the length of delay occurring for each of these phases. Systematic reviews of delay in upper gastrointestinal cancer diagnosis and colorectal cancer diagnosis indicate patient delays ranging from 7 days to 7.5 months and practitioner delays ranging from 0 to 15 months. ${ }^{11}{ }^{12} \mathrm{~A}$ significant issue in studies is how 'delay' is defined and assessed making comparisons between studies problematical. The symptom to diagnosis interval has been used to explore the relationship between delay, stage at 
diagnosis and survival, demonstrating a weak and cancer sitespecific relationship between this interval and survival ${ }^{13}$; however, the definition and measurement of symptom onset is problematical, often leading to inconclusive findings. ${ }^{13}{ }^{14}$ It is also unclear how patient factors such as behavioural responses to symptoms may interact with the biological characteristics of tumours. ${ }^{15} 16$ The development of referral guidelines and performance management strategies for system-generated waiting times has emphasised the importance of practitioner and system delay. ${ }^{17}{ }^{18}$ However, studies indicate that patient delay and in particular 'appraisal delay', which is the period of time during which an individual is trying to determine the cause and significance of their symptoms, may account for the greatest proportion of delay. ${ }^{18-22}$

Factors that contribute to patient delay have been identified, such as low symptom awareness and knowledge of the disease, failure to interpret symptoms as problematical, incorrect selfdiagnosis and self-medication. ${ }^{23}$ The nature of the symptom itself has been identified as important in relation to the timing of diagnosis, and is likely to influence both the timing of presentation and professionals' interpretations of symptoms. For example, even though patients presenting to emergency services with cancer of the digestive tract have been found to report a shorter duration of symptoms, less specific symptoms and fewer visits to physicians than non-emergency admissions, most had made repeated visits to a doctor before being admitted, indicating delay at the primary care level. ${ }^{14}$ Serious symptoms, sometimes referred to as 'alarm' symptoms, are more likely to prompt early diagnosis than vague or commonly experienced symptoms. ${ }^{24}$ Co-morbidities or multiple symptoms can promote shorter delay as these increase concern for individuals; however, they can also inhibit diagnosis if they lead to the misinterpretation or masking of the symptoms of cancer. $^{12} 25$

Systematic review evidence provides insights into the body of work examining delay in cancer diagnosis. The focus has largely been on establishing that delay occurs, and identifying factors that correlate with delay. Such reviews have tended to demonstrate that the burden of delay primarily rests with individuals who do not recognise symptoms in themselves as serious or warranting a visit to the doctor. ${ }^{23}$ Factors such as gender, increasing age and lower socioeconomic status, distance from a cancer centre and lower levels of education have variously been found to contribute to delay, although not consistently, and thus timely diagnosis appears to have a strong socially determined element to it. These factors are also associated with the way individuals access hospital care for cancer. ${ }^{26}$ Methodological differences might be blamed for inconsistencies in findings between studies of patient delay. ${ }^{27}$ However, overcoming such differences will not in itself provide explanations for correlations between 'factors' such as socioeconomic circumstance and delay, or provide deeper understanding of the social processes through which these relationships are produced. Little work has been undertaken to establish why and how delay occurs.

The suggestion that delay may be an inappropriate term is supported by concepts arising out of the medical sociology literature and empirical studies that indicate late cancer diagnosis does not always arise through a failure to act on symptoms experienced by individuals. These studies have explained 'late diagnosis' in relation to the ways in which changes in health are interpreted by those experiencing them before their recognition as 'symptoms', and suggest that the concept of delay fails to address the complex factors at play in how individuals come to recognise that they are ill (J Moffat, PhD thesis, 2007). ${ }^{22} 2528$

\section{CANCER AWARENESS}

Knowledge of symptoms that might indicate cancer is recognised as a prerequisite for early help-seeking and data indicate that there is a low level of community awareness of some symptoms of cancer as well as what to do or how to act if symptoms arise. ${ }^{29}$ Awareness of symptoms as a sign of cancer has been found to be low in particular groups who also have worse health outcomes, such as the socially and economically deprived, men and people from minority ethnic groups. ${ }^{29} \mathrm{~A}$ national cancer awareness measure has been developed and is to be used to monitor awareness of cancer in the UK population over time. Interventions to raise awareness have been the mainstay of public health intervention programmes. Most evaluated interventions to raise awareness of cancer have been targeted at increasing individual knowledge rather than engaging whole communities or social systems. ${ }^{30}$ When communities have been involved or targeted, evaluation tends to have been limited. A healthy communities collaborative approach has been advocated by the Department of Health for England to improve cancer awareness and tackle late cancer diagnosis. ${ }^{31}$ This 'community led' approach enables community groups to generate interventions to address health problems within their local area. $^{32}$ At the time of the NAEDI launch in 2009 the approach was being used to promote cancer awareness in 19 primary care trusts across England. Evaluations of these programmes, using methods traditionally used to evaluate public health interventions, tend to be underpowered to detect statistically significant improvements in early diagnosis. Identifying which components of complex interventions are effective also often proves to be problematical. To date limited evidence exists to evaluate the way such programmes may or may not be translated into behaviour by individuals. Austoker et al, ${ }^{30}$ in a review of the research literature, found insufficient evidence that interventions delivered to individuals promoted early presentation and limited evidence that interventions delivered to communities promote cancer awareness and early presentation. Whereas underpowered studies and service evaluation reports linked to community collaborative type projects are less likely to be published in the research literature, making their systematic evaluation difficult, a number of community intervention projects that indicate some success can be identified. For example, the Early Lung Cancer Intervention in Doncaster project (ELCID) used a social marketing approach based upon a public awareness campaign and an intervention aimed at general practitioners about the benefits of early lung cancer diagnosis. The stage of diagnosis changed pre and post-campaign from $11 \%$ (stages I and II) to 19\%, providing indirect evidence of the impact of the campaign $(p<0.02) .{ }^{33}$

\section{HELP-SEEKING BEHAVIOUR}

A parallel strand of work has developed and tested theory to account for factors that promote or inhibit help-seeking behaviour. A number of theoretical frameworks has been proposed to explain an individual's predisposition to act on symptoms or health changes they may experience. These primarily psychological theories draw on concepts such as reasoned action and planned behaviour, as well as self-regulation theory, to explain and predict the individual tendency to act on symptoms by seeking medical assistance. ${ }^{34}$ For example, Bish et al ${ }^{35}$ develop a theoretical model incorporating stages of symptom appraisal, attitudes towards help-seeking and translating intentions into behaviour, to explain delayed help-seeking. Whereas these psychological models claim to identify psychological processes and attributes of individuals that explain help-seeking, they do 
not explain the social processes and relationships within which help-seeking takes place. In contrast, a metasynthesis of qualitative studies of patients' experiences of help-seeking and delay in cancer diagnosis ${ }^{36}$ found the main reasons for delay in helpseeking to be difficulties in recognising and interpreting symptoms, fear of cancer and embarrassment, and gender-related issues inhibiting help-seeking. The process of sanctioning-that is, those processes that legitimate action-appeared important, such as family members or friends encouraging or forcing contact with health professionals; conversely, reaching crisis point with symptoms legitimises contact with emergency services. Also important is the process by which individuals reveal symptoms to doctors. For example, if there is uncertainty about whether a particular symptom or problem poses a legitimate reason to seek medical help, individuals may instead reveal symptoms while consulting for another problem.

\section{DEVELOPING A BROADER RESEARCH AGENDA}

The drive to develop new approaches to promoting earlier symptomatic diagnosis as an adjunct to cancer screening programmes is a new and emergent science. It is clear that there is significant potential to improve stage at diagnosis by addressing the problem of late presentation and the concerted effort underway should in time bear fruit. There is a growing body of evidence on which to draw; however, a set of rather narrow channels has been pursued to date. The focus has largely been on the individual's response to symptoms or bodily changes they experience, or on professionals and professional systems for diagnosing cancer in isolation from the wider sociocultural context in which ideas about health, illness and responses to illness are formed and enacted. Theoretical approaches to the problem of late diagnosis remain firmly within the science of the psychology of the individual or psychosocial frames of reference. These approaches tend to assume that delay is entirely determined through individual decisions, judgements and behaviours, and does not then take into consideration the wider contextual influences such as living in a rural area or the social context in which ideas about illness, illness causation and health system responses to illness are constructed. Social factors are considered, but only in as far as there may be individual characteristics that have a bearing on behaviour such as educational or socioeconomic background. Interventions to promote earlier diagnosis or prevent late diagnosis drawn from this perspective are unlikely to promote lasting or significant change because the environment in which individual behaviour is shaped, and the ways in which this environment varies across social and cultural groups, is not addressed. Therefore we recommend the development of a third strand of research, which focuses on the broader social dimensions and processes that may impact on the timing of diagnosis.

\section{PATIENT-HEALTH PROFESSIONAL INTERACTIONS}

The intersection of lay conceptions of health and illness with those of practitioners and health systems is important. Views of health and ill health vary across social groups, cultures and circumstances. $^{28} 3738$ Symptoms as they are presented in information aimed at the public regarding awareness of symptoms that might indicate cancer, are presented within a biomedical frame of reference, as are the approaches to the detection of these during medical assessment. Yet there is evidence that lay views of health, symptoms and bodily changes are experienced and expressed very differently to the way in which medical professionals approach assessing the signs and symptoms of ill health of individuals presenting in medical encounters. Individuals focus more on personal practices and responsibilities and this often has a strong moral dimension relating to what one 'should' do to maintain health and practices that will ward off ill health or help one return to full health. ${ }^{39}$ For some, poor health is seen to be due to 'wrong' living. 'Keeping going' and not letting things go are important drivers underlying lay views of health. In the context of attempting to speed the recognition of ill health that might indicate cancer, these lay conceptions of health may act as barriers to presentation. They may also conflict with the health professional's diagnostic agenda, further complicating the diagnostic process. Perspectives that draw on an extensive literature relating to social dimensions of health and illness have yet to be applied in studies in the context of cancer. Furthermore, improved understandings of how lay views and consulting behaviour differ by socioeconomic group would inform interventions to improve cancer survival and thus address the socioeconomic gradient in health outcomes.

A number of studies of the process of 'becoming diagnosed' with cancer has highlighted important social dimensions in the way in which individuals respond to the possibility of ill health. Shame and stigma are described as a core component of individual responses to a diagnosis of lung cancer. For example, the belief that health professionals will view an individual who smokes and presents with chest-related symptoms as at fault because of the association of lung cancer with smoking may make individuals feel reluctant to seek help. ${ }^{40}$ Furthermore, the identification of social sanctioning (ie, the role played by lay networks, health services and wider society in supporting and prompting help-seeking) as an important factor in recent systematic reviews, draws attention to the social nature of individuals' recognition of their own illness. ${ }^{23}$ If, as Radley and Billig $^{41}$ theorise, illness is viewed as an experience or condition that can only be sustained in one's mind as a possibility 'against the background of other competing moral demands', it does not appear surprising that the social sanctioning of ill health is implicated in illness recognition as well as help-seeking. ${ }^{42}$

Understanding of the ways in which illness recognition can be socially determined is emerging and suggests that a linear view of illness recognition and help-seeking in which a person experiences a symptom, recognises it as such and then presents it to a primary care physician is too simplistic. When faced with multiple possibilities for socially legitimate non-disease explanations for changes in one's health, and a need to legitimise claims to ill health (L Brindle, $\mathrm{PhD}$ thesis, 2000), ${ }^{43}{ }^{45}$ the interpretation of a change in health as illness, or a symptom, is evidently a socially mediated (or constructed) process. ${ }^{44} 45$ From a perspective that treats symptom recognition as a social process, the attribution of delay in presentation to individuals and, therefore, a clear distinction between patient and health practitioner 'delay', becomes problematical. ${ }^{42} 4546$

Arguments such as these from medical sociology, and a growing body of empirical research that demonstrates how the micro-organisation of primary care consultations and the terminology used by clinicians influences clinical outcomes, support an argument for adopting research approaches such as ethnography and ethnomethodology. These approaches set out to understand how ill health becomes recognised as such within social interactions. Such research involves the sociological analyses of observations and recordings of naturally occurring interactions between patients and health professionals, or patients and family members, and focuses on the factors that influence how individuals interpret and act in relation to their 
health and symptoms as observed within everyday interactions. These approaches have the potential to inform the development of interventions to improve earlier presentation and earlier referral for investigation of symptoms. For example, the use of an ethnomethodological approach has been proposed to improve earlier lung cancer diagnosis by developing targeted interventions to improve the elicitation of health changes in routine clinical consultations involving those at increased risk. ${ }^{42}$

Other studies reveal difficulties for individuals in convincing doctors that that their symptoms are serious and this reinforces a developing literature relating to the complexity surrounding help-seeking encounters with professionals. On the one hand there is enormous difficulty for professionals in interpreting often vague and commonly experienced symptoms to determine which warrant referral for further investigation, and for individuals on the other hand, the challenge of being 'convincing' or presenting one's problem in a way that will fit with a doctor's biomedical frame of reference. ${ }^{47} 48$

The identification of cancer-specific relationships between risk factors and delayed diagnosis in systematic reviews of delay might reflect the differing narratives associated with different types of cancer. Moffat (Moffat J. Understanding the experience of developing and being diagnosed with lung cancer: exploring the potential of narrative. Unpublished $\mathrm{PhD}$ Thesis. University of Southampton, 2007) suggests that the absence of a narrative of lung cancer; a story or plot in which an individual can place one's self may be a part of why individuals delay presenting to their doctor with symptoms that are subsequently found to be caused by lung cancer. Corner et $a l^{22}$ reveal the difficulty for individuals in interpreting bodily changes that are a prelude to lung cancer as anything more than an inevitable part of getting older and therefore to be expected. Tishelman and Sachs ${ }^{49}$ describe this process among women with cervical cancer as a 'subtle normalising experience' that proceeds over time in which symptoms may be legitimised and non-legitimised through encounters with medical professionals. Andersen et al ${ }^{44}$ reveal how individuals 'contain' or minimise bodily sensations by integrating these into culturally acceptable explanations of bodily changes such as 'heavy garden work' or stress. They may also disassociate themselves from lifestyles that might put themselves at risk of cancer or other serious disease and, thus, disregard disease explanations for ill health. A sense of obligation to maintain normality allows individuals to put off interpreting symptoms as requiring health care or investigation. The experience of 'embodied' ill health (ie, the physical sensation of ill health) ${ }^{50}$ may be lacking for some cancers, not necessarily because there are no symptoms, but because such symptoms or bodily changes are not constructed socially into a commonly understood frame of reference for individuals so that they can be recognised and how one is meant to act in relation to them widely understood. The social understanding surrounding a breast lump and what one should do about it, for example, has now largely become a socially understood condition as a result of media and public health messages. This is not the case for many other cancers.

Further to the complexities of what prompts help-seeking and the challenges surrounding individual-professional interactions in recognising signs of ill health and appropriate and timely action being taken by doctors, there are indications that the wider social environment surrounding particular diseases may also be important. Corner et al ${ }^{22}$ have suggested that a social narrative that relates early symptomatic diagnosis to improved survival has not as yet been constructed around diseases such as lung cancer, and outlines the elements of what needs to be present for the 'social discovery' of disease. ${ }^{51}$ These include lay initiation in seeking help, a social movement demanding action, professional agendas to champion the disease and strong organisational or institutional backing. In the absence of these it is argued that it is unlikely that a strong or effective environment for the identification and management of a particular illness condition is likely to be mobilised.

\section{SOCIAL PROCESSES AND PROMOTING EARLIER DIAGNOSIS OF CANCER: THE RESEARCH AGENDA}

The contribution of individual behaviour to late presentation and diagnosis of cancer and the social processes that create this phenomenon is only now beginning to be recognised as worthy of study. This is not least because evidence that avoidable, late diagnosis is a significant factor in cancer survival outcomes, even in cancers in which there is a well-organised national screening programme with high participation, is only just emerging.

There is increasing evidence that avoidable 'delays' before diagnosis are a significant factor in cancer survival outcomes. Attempts to isolate individual from social, and primary care physician from patient factors, have led to the identification of inconsistent relationships between 'social factors' and diagnostic delay, with little explanation for these apparent relationships. We suggest a new research agenda is required that sets out to study in detail the processes by which individuals come to recognise and act on health changes as a prelude to a cancer diagnosis and how recognition occurs within, rather than independently of, or in interaction with, a social context. This

\section{What is already known on this subject}

Avoidable delay in diagnosis is a significant factor in cancer survival outcomes. Individuals may contribute to delayed diagnosis through not recognising symptoms or health changes that are related to cancer or not seeking help for these promptly.

\section{What this paper adds}

The processes by which patients seek help for health changes that might be symptoms of cancer and secure diagnostic investigations is complex and do not relate solely to individual patient or professional behaviours. The wider societal context in which illness and disease is shaped and defined through medical knowledge, belief systems and organisations is also important. Social processes shape how symptoms are interpreted and presented to doctors in consultations; these processes warrant further exploration and investigation.

\section{Policy implications}

Research addressing the social processes involved in the timing of diagnosis of cancer, and public health interventions informed by this research, are urgently needed to address the problem of late diagnosis. 
demands studies that employ contextually sensitive methods using observational and participatory methods such as ethnography or ethnomethodology. ${ }^{52-54}$ Here social action is observed in situ as events arise in order to develop deep contextually derived knowledge of the problem of late diagnosis. The findings of detailed contextually situated insights into what promotes and inhibits help-seeking seems a fruitful starting point for developing and testing community-based interventions based on whole systems thinking.

Insights derived through research developed through this perspective could be applied in a variety of ways, for example: community-based awareness programmes based on deep knowledge of how symptoms are interpreted in everyday situations among particular communities and groups; re-engineering health system pathways for diagnosis; the development of innovative diagnostic decision tools that recognise the complexities of symptom presentation; and 'lay'-health practitioner communication about early symptoms; as well as population-based studies to elucidate risk factors for cancer that incorporate understanding derived through the study of social processes. This research agenda fits within the 'new' approach to public health research advocated by authors such as Paterson ${ }^{55}$ and Popay and Williams, ${ }^{56}$ who argue that epidemiology relies too heavily on population data and survey work to develop an understanding of patterns of disease while neglecting the social dynamics involved in the epidemiology of disease. They suggest that a powerful and untapped resource for understanding the connection between social dynamics and ill health is held among individuals and people who are ill. $^{56}$ We believe that new, innovative approaches to the pressing public health problem of late diagnosis in cancer are waiting to be discovered through the application of knowledge derived from 'bottom-up' observation and investigation with people and communities that could simultaneously be applied to the development and testing of public health interventions.

Competing interests None.

Provenance and peer review Commissioned; externally peer reviewed.

\section{REFERENCES}

1. Department of Health. Cancer reform strategy, 2nd annual report. London: Department of Health, 2009.

2. Department of Health. National cancer equality initiative report. London: Department of Health, 2010

3. Berrino F, De AR, Sant M, et al. Survival for eight major cancers and all cancers combined for European adults diagnosed in 1995-1999: results of the EUROCARE 4 study. Lancet Oncol 2007:8:773-83.

4. Sant $\mathbf{M}$, Allemani C, Capocaccia R, et al. Stage at diagnosis is a key explanation of differences in breast cancer survival across Europe. Int J Cancer 2003;106:416-22.

5. Richards $\mathbf{M}$. The national awareness and early diagnosis initiative in England: assembling the evidence. Br J Cancer 2009;101(Suppl 2):S1-4.

6. Møller $\mathbf{H}$, Linklater KM, Robinson D. A visual summary of the EUROCARE 4 results: a UK perspective. Br J Cancer 2009;101(Suppl 2):S110-14.

7. Thomson CS, Forman D. Cancer survival in England and the influence of early diagnosis: what can we learn from recent EUROCARE results? Br J Cancer 2009;101 (Suppl 2):S102-9.

8. Hamilton W. The CAPER studies: five case-control studies aimed at identifying and quantifying the risk of cancer in symptomatic primary care patients. $\mathrm{Br} \mathrm{J}$ Cancer 2009;101 (Suppl 2):S80-6.

9. Burgess C, Ramirez AJ, Richards MA, et al. Who and what influences delay among women reporting symptoms of breast cancer? Br J Cancer 1998;71:1343-8.

10. Nichols S, Waters WE, Fraser JD, et al. Delay in the presentation of breast symptoms for consultant investigation. Community Med 1981;3:217-25.

11. Macdonald S, Macleod U, Campbell NC, et al. Systematic review of factors influencing patient and practitioner delay in diagnosis of upper gastrointestinal cancer. Br J Cancer 2006:94:1272-80.

12. Mitchell E, Macdonald S, Campbell NC, et al. Influences on pre-hospital delay in the diagnosis of colorectal cancer: a systematic review. Br J Cancer 2008:98:60-70.

13. Maguire A, Porta M, Malats N, et al. Cancer survival and the duration of symptoms. An analysis of possible forms of the risk function. Eur J Cancer 1994;30A:785-92.
14. Porta M, Fernandez E, Belloc J, et al. Emergency admission for cancer: a matter of survival? Br J Cancer 1998;77:477-84.

15. Porta M, Gallen M, Malats N, et al. Influence of "diagnostic delay" upon cancer survival: an analysis of five tumour sites. J Epidemiol Community Health 1991:45:225-30

16. Fernandez E, Porta M, Malats $\mathrm{N}$, et al. Symptom to diagnosis interval and survival in cancers of the digestive tract. Digest Dis Sci 2002;47:2434-40.

17. Department of Health. The cancer plan. London: Department of Health, 2000.

18. Department of Health. Referral guidelines for suspected cancers. London: Department of Health, 2000.

19. Anderson BI, Cacioppo JT, Roberst DC. Delay in seeking a cancer diaognosis: delay stages and psychological comparison processes. Br J Soc Psychol 1995:34:33-52.

20. Ristvedt SL, Trinkaus KM. Psychological factors related to delay in consultation for cancer symptoms. Psycho-oncol 2004;14:339-50.

21. Corner $\mathbf{J}$, Hopkinson J, Fitzsimmons D, et al. Is late diagnosis of lung cancer inevitable? Interview study of patients' recollections of symptoms before diagnosis. Thorax 2005:60:314-19.

22. Corner J, Hopkinson J, Roffe L. Experience of health changes and reasons for delay in seeking care: a UK study of the months prior to the diagnosis of lung cancer. Soc Sci Med 2006;62:1382-91.

23. Macleod U, Mitchell ED, Burgess C, et al. Risk factors for delayed presentation and referral of symptomatic cancer: evidence for common cancers. Br J Cancer 2009;101 (Suppl 2):S92-101.

24. Jones R, Latinovic R, Charlton J, et al. Alarm symptoms in early diagnosis of cance in primary care: cohort study using general practice research database. BMJ 2009; 339:b3094.

25. Lovgren M, Levealahti $\mathrm{H}$, Tishelman $\mathrm{C}$, et al. Time spans from first symptoms to treatment in patients with lung cancer-the influence of symptoms and demographic characteristics. Acta Oncol 2008;47:397-405.

26. Raine R, Wong W, Scholes S, et al. Social variations in access to hospital care for patients with colorectal, breast and lung cancer between 1999 and 2006: retrospective analysis of hospital episode statistics. BMJ 2010;340:b5479.

27. Neal RD, Pasterfield D, Wilkinson C, et al. Determining patient and primary care delay in the diagnosis of cancer-lessons from a pilot study of patients referred for suspected cancer. BMC Fam Pract 2008;9:9

28. Good BJ, Good MD. The meaning of symptoms: a cultural hermeneutic model for clinical practice. In: Eisenberg L, Kleinman A, eds. The relevance of social science for medicine. Holland: D Reidel Publishing Company, 1981:165-96.

29. Robb K, Stubbings $\mathrm{S}$, Ramirez A, et al. Public awareness of cancer in Britain: a population-based survey. Br J Cancer 2009:101(Suppl 2):S18-23.

30. Austoker L, Bankhead C, Forbes LJL, et al. Interventions to promote cancer awareness and early presentation: systematic review. $\mathrm{Br} J$ Cancer 2009;101 (Suppl 2):S31-9.

31. Improvement Foundation. The Healthy Communities Collaborative: new partnerships to tackle inequalities. Improvement Foundation 2007.

32. Lyon D, Knowles J, Slater B, et al. Improving early presentation of cancer symptoms in disadvantaged communities: putting local people in control. $\mathrm{Br} \mathrm{J}$ Cancer 2009;101(Suppl 2):S49-54.

33. Suckling R. Early lung cancer intervention in Doncaster (ELCID) project report for NHS Doncaster primary care trust. 2008.

34. O'Mahony M, Hegarty J. Help seeking for cancer symptoms: a review of literature Oncol Nurs Forum 2009;36:E178-84.

35. Bish A, Ramirez A, Burgess $\mathrm{C}$, et al. Understanding why women delay in seeking for help for breast symptoms. J Psychosom Res 2005;58:321-6.

36. Smith LK, Pope C, Botha JL. Patients' help-seeking experiences and delay in cancer presentation: a qualitative synthesis. Lancet 2005;366:825-31.

37. Twaddle AC. Sickness and the sickness career: some implications. In: Eisenberg $L$, Kleinman A, eds. The relevance of social science for medicine. Holland: D Reidel Publishing Company, 1981:111-33.

38. Zola IK. Culture and symptoms: an analysis of patients' presenting complaints. In: Cox C, Mead A, eds. A sociology of medical practice. London: Collier-Macmillan, 1975:23-348.

39. Shaw Hughner $\mathbf{R}$, Schultz Kleine S. Views of health in the lay sector: a complilation and review of how individuals think about health. Health 2004;8:395-422.

40. Chapple A, Ziebland S, McPherson A. Stigma, shame and blame experienced by patients with lung cancer: qualitative study. BMJ 2004;328:1470-5.

41. Radley A, Billig M. accounts of health and illness: dilemmas and representations. Sociol Health III 1996;18:220-40.

42. Brindle L. Deconstructing symptoms: developing an ethnomethodological understanding of 'patient delay' in lung cancer diagnosis. In: Proceedings of the British Sociological Association Medical Sociology Conference; September 2007. Liverpool, UK: British Sociological Association, 2007:54.

43. Brindle L. Narratives of health protection in families with a late-onset kidney disease: re-defining governmentality and responsibility for health in the era of the 'new' genetics. Special edition of sociological research online: big structures, large processes, huge comparisons-narratives from minor to major. 2009;14:23. 2060. http://www.socresonline.org.uk/14/5/23.html (accessed 1 Sep 2010).

44. Andersen RS, Paarup B, Vedsted P, et al. 'Containment' as an analytical framework for understanding patient delay: a qualitative study of cancer patients' symptom interpretation processes. Soc Sci Med 2010;71:378-85.

45. Robinson JD, Heritage J. The structure of patients' presenting concerns: the completion relevance of current symptoms. Soc Sci Med 2005;61:481-93. 
46. Heritage J, Robinson JD, Elliot MN, et al. Reducing patients' unmet concerns: the difference one word can make. J Gen Intern Med

2007;22:1429-33.

47. Stivers T. Treatment decisions: negotiations between doctors and patients in acute care encounters. In: Heritage J, Maynard D, eds. Communication in medical care: interaction between primary care physicians and patients. Cambridge (UK): Cambridge University Press, 2006:279-312

48. Collins S, Britten N, Ruusovouri, et al. Understanding the process of patient participation. In: Collins S, Britten N, Ruusovouri J, et al, eds. Patient participation in health care consultations: qualitative perspectives. Maidenhead: McGraw-Hill/Open University Press, 2007:3-21.

49. Tishelman C, Sachs L. The diagnostic process and the boundaries of normality. Qual Health Res 1998;8:48-60.
50. Lawton J. Colonising the future: temporal perceptions and health-relevant behaviours across the adult lifecourse. Sociol Health III 2002;24:714-33.

51. Brown P. Naming and framing: the social construction of diagnosis and illness. J Health Soc Behav 1995;35:34-52.

52. Silverman D. Harvey Sacks: Social science and conversation analysis. Cambridge: Polity Press, 1998:1-232

53. Garfinkel H. Studies in ethnomethodology. Englewood Cliffs: Prentice-Hall, 1967:1-304.

54. Hammersley M, Atkinson P. Ethnography: principles in practice. London: Tavistock Publications, 1983:1-273.

55. Paterson K. Theoretical perspectives in epidemiology—a critical appraisal. Radical Community Med 1981;23:34

56. Popay J, Williams G. Public health research and lay knowledge. Soc Sci Med 1996;42:759-68 